\title{
Video Article \\ Micro-Mechanical Characterization of Lung Tissue Using Atomic Force Microscopy
}

\author{
Fei Liu ${ }^{1}$, Daniel J. Tschumperlin ${ }^{1}$ \\ ${ }^{1}$ Molecular and Integrative Physiological Sciences, Department of Environmental Health, Harvard School of Public Health
}

Correspondence to: Daniel J. Tschumperlin at dtschump@hsph.harvard.edu

URL: https://www.jove.com/video/2911

DOI: doi:10.3791/2911

Keywords: Biophysics, Issue 54, Atomic force microscopy, indentation, stiffness, fibrosis, extracellular matrix

Date Published: 8/28/2011

Citation: Liu, F., Tschumperlin, D.J. Micro-Mechanical Characterization of Lung Tissue Using Atomic Force Microscopy. J. Vis. Exp. (54), e2911, doi:10.3791/2911 (2011).

\section{Abstract}

Matrix stiffness strongly influences growth, differentiation and function of adherent cells ${ }^{1-3}$. On the macro scale the stiffness of tissues and organs within the human body span several orders of magnitude ${ }^{4}$. Much less is known about how stiffness varies spatially within tissues, and what the scope and spatial scale of stiffness changes are in disease processes that result in tissue remodeling. To better understand how changes in matrix stiffness contribute to cellular physiology in health and disease, measurements of tissue stiffness obtained at a spatial scale relevant to resident cells are needed. This is particularly true for the lung, a highly compliant and elastic tissue in which matrix remodeling is a prominent feature in diseases such as asthma, emphysema, hypertension and fibrosis. To characterize the local mechanical environment of lung parenchyma at a spatial scale relevant to resident cells, we have developed methods to directly measure the local elastic properties of fresh murine lung tissue using atomic force microscopy (AFM) microindentation. With appropriate choice of AFM indentor, cantilever, and indentation depth, these methods allow measurements of local tissue shear modulus in parallel with phase contrast and fluorescence imaging of the region of interest. Systematic sampling of tissue strips provides maps of tissue mechanical properties that reveal local spatial variations in shear modulus. Correlations between mechanical properties and underlying anatomical and pathological features illustrate how stiffness varies with matrix deposition in fibrosis. These methods can be extended to other soft tissues and disease processes to reveal how local tissue mechanical properties vary across space and disease progression.

\section{Video Link}

The video component of this article can be found at https://www.jove.com/video/2911/

Protocol

\section{Lung Tissue Strip Preparation}

1. Lung tissue is highly compliant and difficult to cut into strips for AFM characterization. To transiently stabilize the lung structure for cutting, inflate isolated mouse lungs intratracheally with $50 \mathrm{ml} / \mathrm{kg}$ body weight of $2 \%$ low gel point agarose (prepared in PBS) warmed to $37^{\circ} \mathrm{C}$. Tie off the trachea and cool the inflated lungs in a bath of PBS at $4^{\circ} \mathrm{C}$ for 60 minutes. The agarose will gel and stiffen in the airspaces to gently stabilize the lung structure during this interval ${ }^{5}$. Higher agarose concentrations, i.e. $3-4 \%$, may be used to further enhance tissue stabilization for cutting.

2. Cut the agarose-stabilized mouse lung tissue with a razor or scalpel blade into strips of $5 \times 5 \mathrm{~mm}$ in length and width and $400 \mu \mathrm{m}$ in thickness, then wash the strips in a PBS bath pre-warmed to $37^{\circ} \mathrm{C}$ using a $100 \mathrm{ml}$ glass beaker on a bench-top heated stir plate for 5 minutes to remove residual agarose. To exclude large airways and vessels, cut strips from subpleural regions distant from main stem bronchi. If airways and large vessels are to be imaged, cut strips from lung tissue more proximal to main stem bronchi.

\section{AFM Microindentation and Fluorescence Imaging}

1. To isolate areas of interest for AFM microindentation, tissue can be visualized by phase contrast microscopy, or immunostained and visualized by fluorescence microscopy. For immunostaining, block tissue strips with $5 \%$ serum from the source of the desired secondary antibody (in PBS) for 2 hours without fixation or permeabilization at room temperature.

2. Incubate the tissue strip with appropriate primary antibody (e.g. rabbit anti-collagen I to visualize interstitial collagen (1:250 dilution), rabbit anti-laminin (1:250 dilution) to visualize basement membrane) in a well of a 12-well plate on a rocker with gentle shaking for 1 hour, wash sample 3 times for 5 minutes each with PBS, followed by appropriate secondary antibody conjugated to fluorescent tag (e.g. Alexa Fluor 546 goat anti-rabbit secondary antibody (1:250 dilution)) for 1 hour at room temperature.

3. Wash tissue strips extensively with PBS and store in PBS at $4{ }^{\circ} \mathrm{C}$

4. Immediately before AFM characterization, attach the tissue strip to a poly-l-lysine-coated 15-mm coverslip by lifting the coverslip from below the floating tissue, making sure the tissue strip spreads evenly on the coverslip surface; if necessary, sandwich the strip with a second clean, uncoated coverslip and apply mild pressure to assist with tissue attachment to the poly-I-lysine-coated coverslip. 
5. Calibrate the AFM system following the manufacture's instruction immediately before each round of microindentation experiments. Determine two critical parameters: 1) the cantilever spring constant using the thermal fluctuation method in air ${ }^{6}$; and, 2) the cantilever deflection sensitivity, which is a parameter used to scale the photodiode output signal to the actual cantilever deflection distance, $\Delta \mathrm{d}$. Calibrate deflection sensitivity by obtaining a standard force-displacement curve in PBS on a clean glass slide then calculate the slope of the forcedisplacement curve (Fig.1B). In the force-displacement curve measurement, the AFM tip is extended towards and retracted from the sample surface at a single location (without rastering in X-Y directions), with the deflection of the cantilever, $\Delta \mathrm{d}$, monitored as a function of tip displacement, $\Delta \mathbf{z}$.

We recommend using a silicon nitride triangle cantilever with a 5- $\mu \mathrm{m}$ diameter borosilicate spherical tip (Novascan). Using AFM probes with a spring constant of $0.06 \mathrm{~N} / \mathrm{m}$, we have mechanically characterized soft materials with shear moduli spanning 100 to $50,000 \mathrm{~Pa}$.

6. Wipe the bottom surface of the sample coverslip with tissue paper, fasten it to a standard glass slide with vacuum grease, mount the glass slide on the AFM sample stage, and cover the tissue with $500 \mu$ I PBS (room temperature).

7. Set the microscope for eye-piece viewing to align the AFM tip in the center of the field of view by adjusting the microscope sample stage. Choose an area of interest on the tissue by moving the AFM sample stage, then switch to CCD camera viewing to record phase contrast image and/or fluorescence images of the tissue, as desired.

8. Perform standard AFM engagement operation by moving the AFM tip slowly downwards until it is in contact with the sample.

9. Accurate AFM microindentation characterization of soft samples requires small indentation depths to avoid large local strains which invalidate the Hertz model used for elastic modulus calculation. To avoid large strains, perform indentation in trigger mode by setting the cantilever maximum deflection (trigger point) to $500 \mathrm{~nm}$. This deflection limit will restrain the maximum indentation force to less than $30 \mathrm{nN}(\mathrm{F}=$ cantilever spring constant * displacement, or $\mathrm{F}_{\max }=0.06 \mathrm{nN} / \mathrm{nm}{ }^{*} 500 \mathrm{~nm}=30 \mathrm{nN}$ ).

The indentation velocity should be selected to be sufficiently slow to explore elastic rather viscoelastic properties of the soft sample ${ }^{7}$. A velocity range of $2-20 \mu \mathrm{m}$ per second is suitable for lung tissue.

10. For a single measurement from an area of interest, move the AFM probe to the location of interest and perform a single indentation to collect a standard force-displacement curve (the probe moves only in Z-direction without $X-Y$ scanning).

11. For automated mapping of a region of interest, switch to Force-Map mode, select the scan size and sample points within the selected area. In Force-Map mode, the AFM tip rasters across the sample surface between indentation movements, and collects individual force-displacement curves at each point within a defined sample grid. More sampling points will provide higher spatial resolution but lengthen the time required for mapping. We found it practical to use a $16 \times 16$ sample grid to map an $80 \times 80-\mu \mathrm{m}$ area at an indentation velocity of $20 \mu \mathrm{m}$ per second, which can be completed in $\sim 10$ minutes.

\section{AFM Data Extraction}

1. To calculate the Young's modulus, fit the force-displacement curve to the Hertz spherical indentation model using least squares non-linear curving fitting ${ }^{8}$ (Fig.1 A,C):

$$
E=\frac{3\left(1-v^{2}\right) F}{4 R^{1 / 2} \delta^{3 / 2}}
$$

where $F=k_{c}{ }^{*} \Delta d$, is the force to bend the cantilever, $k_{c}$ is the cantilever spring constant, $R$ is sphere tip radius, $\delta=\Delta z-\Delta d$ is indentation and $\mathrm{u}$ is the sample's Poisson ratio $\left(\mathrm{u}=0.4\right.$ for lung tissue $\left.{ }^{9}\right)$.

2. To assess the quality of the fit, calculate the SSR value, or the sum of squares of the difference between the data and the fit values, during the non-linear curve fitting. Eliminate unreliable or un-interpretable measurements by discarding data from "bad" curves with large SSR values.

3. If desired, convert elastic modulus $(E)$ to shear modulus $(G)$, using the relationship $E=2 \times(1+U) \times G$. To visualize spatial patterns of stiffness collected in Force-Map mode, plot modulus data in contour maps (e.g. in $16 \times 16$ sample grid covering an $80 \times 80-\mu \mathrm{m}$ area).

4. To process a large amount of force curve data, a custom algorithm may be written to automatically fit force-displacement curves, extract moduli, and/or plot elastographs using the same procedures and parameters as outlined in 3.2 and 3.3 (e.g. Matlab). 
A

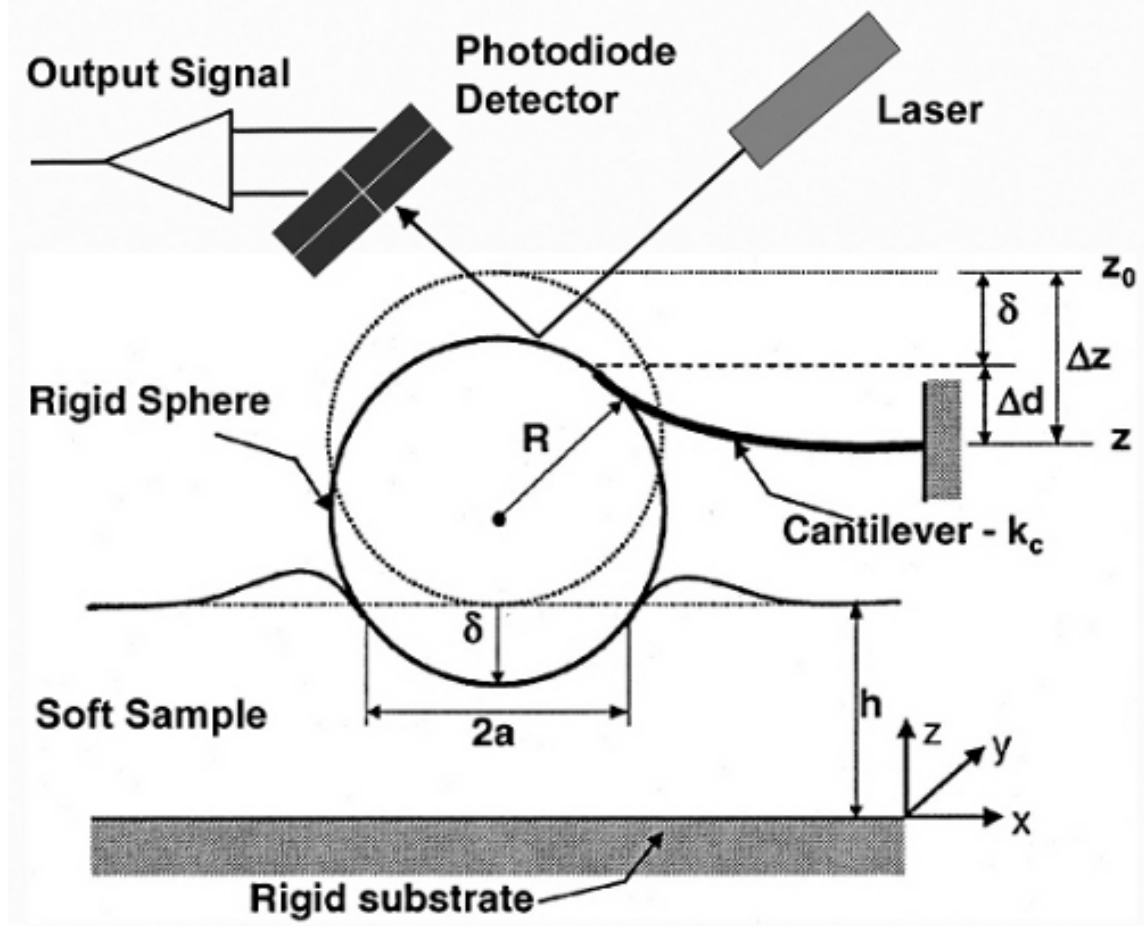

B
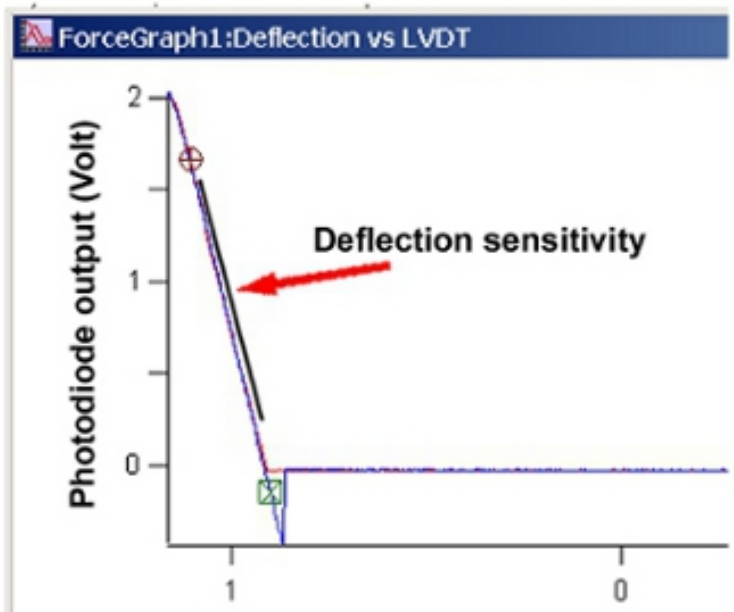

Tip displacement, $\Delta z(\mu \mathrm{m})$

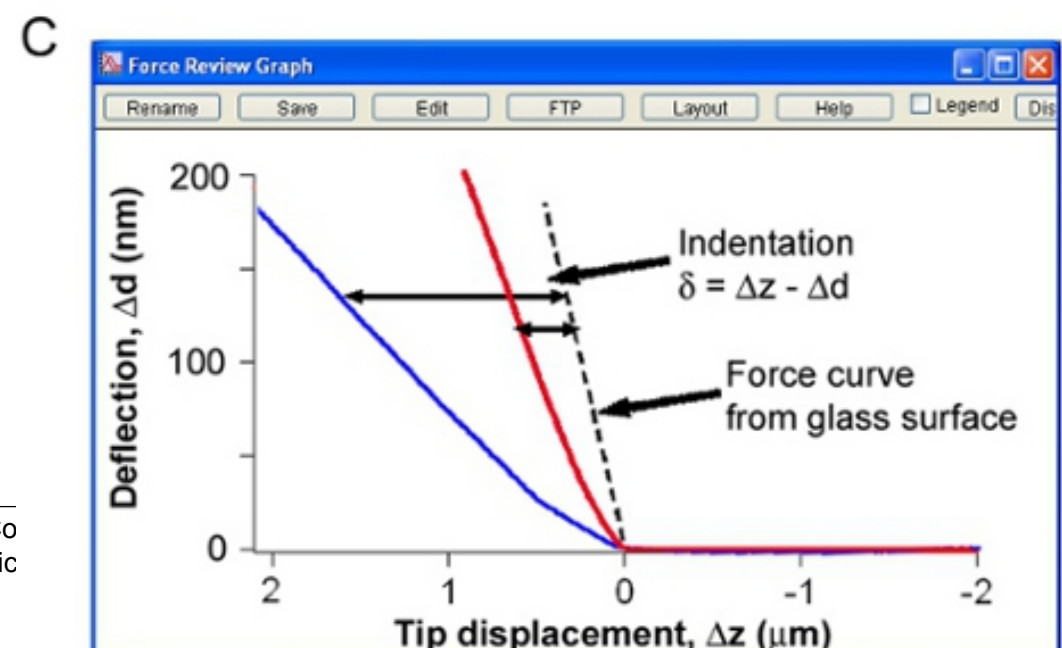


Figure 1 (A) Schematic of AFM microindentation of a soft sample on a rigid substrate using a spherical probe (Reproduced with permission from Dimitriadis E., et al. 2002 Biophys $\mathrm{J}^{8}$ ). (B) Representative force-displacement curve collected from a clean glass slide in PBS to determine cantilever deflection sensitivity. (C) Representative force-displacement curves collected from soft (blue) and stiff (red) samples showing the corresponding parameters from $(A)$.

\section{Representative Results:}

Figure 2A shows a lung parenchyma strip attached on poly-l-lysine coated coverslip in PBS with an AFM probe in place directly above region of interest and ready for microindentation mapping. Figure 2B shows that in properly cut and stained tissue, the alveolar microarchitecture of lung parenchyma is well preserved, as observed by immunofluorescent staining for basement membrane component laminin without fixation or permeabilization. Figure $2 \mathrm{C}$-D show immunofluorescent staining for collagen I in fresh unfixed lungs harvested from mice previously treated with PBS (Fig. 2C), or treated with bleomycin (Fig. 2D) to induce fibrosis ${ }^{10}$.

Figure 3A shows sample force-displacement plots obtained from AFM microindentation. With the same applied force, AFM tip generates a large indentation on a soft region (blue line), resulting in a relatively "flat" force-displacement curve versus a small indentation and a "steep" forcedisplacement curve for a stiffer region (red line). In order to obtain clean force curves, after each indentation the AFM tip needs to be retracted completely from the sample surface and free of contact before the next indentation. This contact free state corresponds to the flat region of the curve in Figure 3A, where the tip translates without cantilever deflection. Figure 3B shows a typical false curve without a flat region which occurs when the tip is not completely retracted from the sample surface (e.g. soft sample attaches to tip) making it impossible to determine the contact point. If the tip is completely trapped in soft sample, the curve may look as shown in Figure $3 \mathrm{C}$, without clear deflection but small noise.

Figure 4 shows shear modulus data extracted from force-displacement curves displayed spatially as stiffness maps (elastographs), where color scales with shear modulus. Elastographs demonstrate striking differences in the range and distribution of tissue shear modulus in normal (Fig. 4A) and fibrotic (Fig. 4B) lung parenchyma, and large spatial variations in shear modulus, particularly within the fibrotic lung sample.
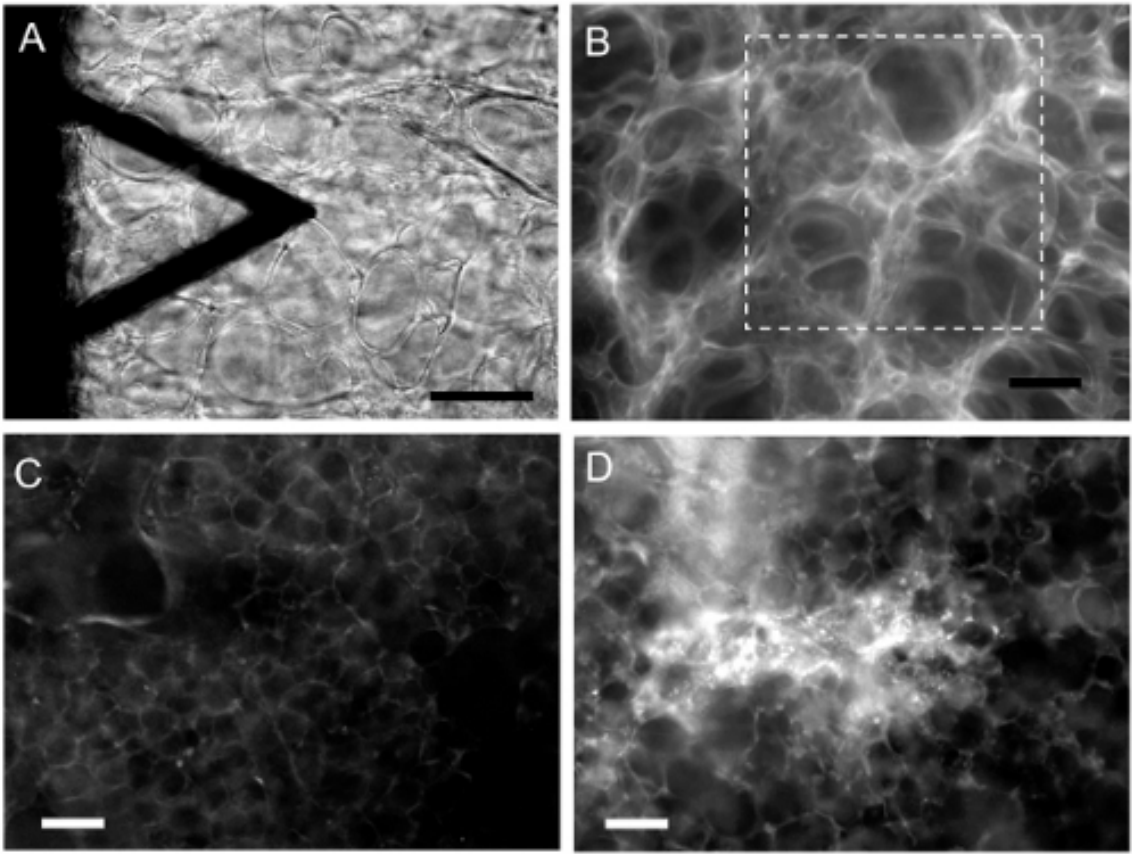

Figure 2. (A) Phase contrast micrograph showing an AFM probe over a mouse lung parenchyma strip. Scale bar, $50 \mu \mathrm{m}$. (B) Immunostaining of laminin in normal mouse lung tissue showing the alveolar microarchitecture of normal lung parenchyma. White box indicates typical 80 by-80 $\mu \mathrm{m}$ elasticity mapping area. Scale bar: $20 \mu \mathrm{m}$. (C and D) Immunostaining of collagen I in saline (C) and bleomycin (D) treated mouse lung parenchyma. Scale bars, $100 \mu \mathrm{m}$. 
A

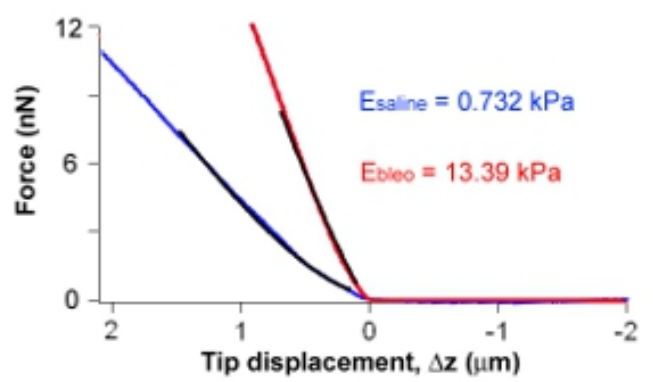

C

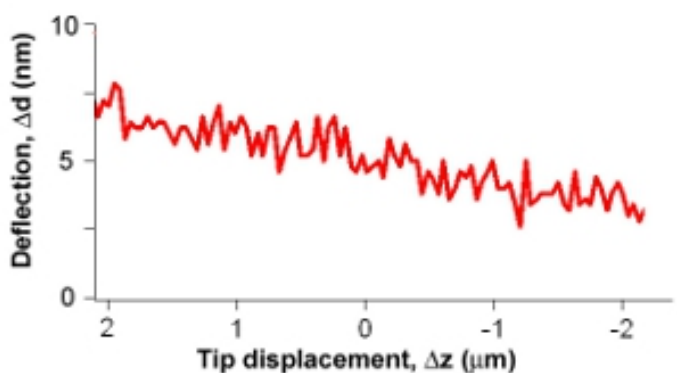

B

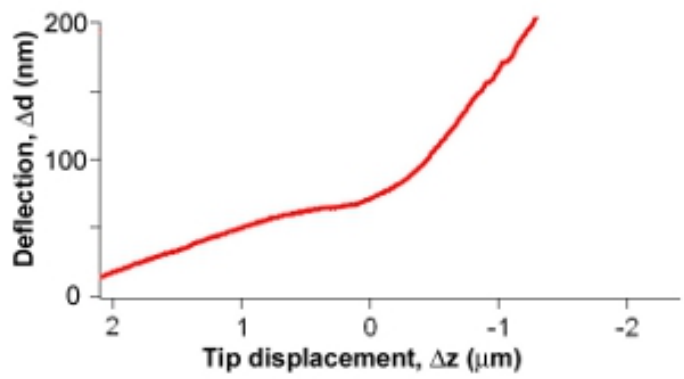

Figure 3. (A) Representative interpretable force-displacement curves collected from saline (blue) and bleomycin-treated (red) mouse lung parenchyma, respectively. Black lines are the best fit resulting from the spherical Hertz model and are labeled with their corresponding calculated Young's moduli. (B, C) Representative un-interpretable force-displacement curves.

A

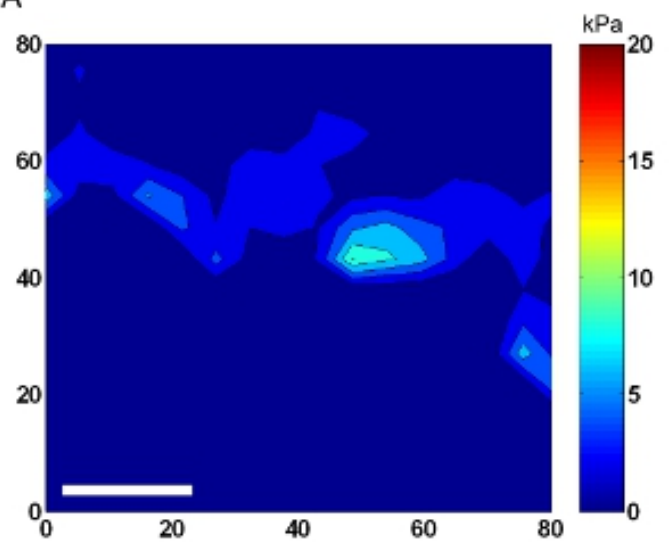

B

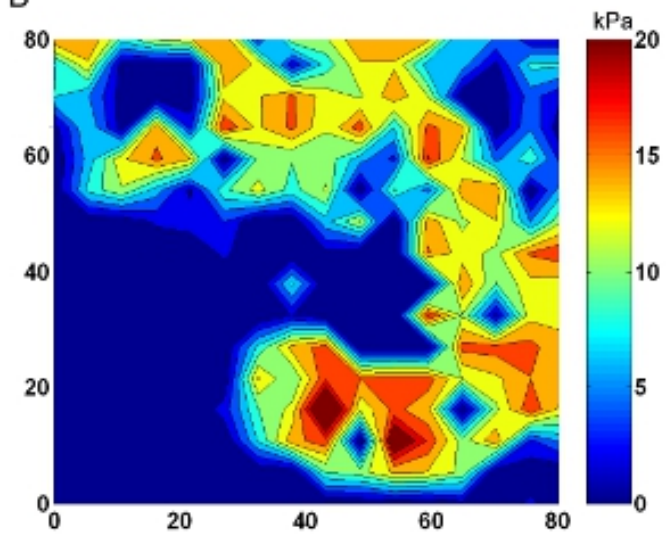

Figure 4. Representative elastographs collected from saline $(A)$ and bleomycin-treated $(B)$ mouse lung parenchyma. The color bars indicate shear modulus in kilopascals. Axis labels indicate spatial scale in micrometers. Scale bar: $20 \mu \mathrm{m}$

\section{Discussion}

Mechanical characterization of lung tissue using AFM microindentation offers unprecedented spatial resolution (Fig. 4), providing a unique perspective on microscale variations in tissue stiffness. As an example of its utility, previous macro-scale measurements in normal and fibrotic lung tissue strips indicated an approximate $2-3$-fold increase in elastance with fibrosis ${ }^{11,12}$. In contrast, AFM microindentation reveals that tissue stiffening is highly localized, with some regions exhibiting up to $\sim 30$-fold increases in shear modulus above the median observed in normal lung tissue ${ }^{10}$. As matrix stiffness is now known to critically influence cell function, these local measurements provide invaluable parameters to enhance the biofidelity of cell culture study of lung cells.

Several practical issues arise with the use of thin strips of lung tissue. The surfaces of the strips are not perfectly flat, as the tissue profile follows the architecture of the underlying alveoli. The AFM system automatically adjusts tip position in the Z-direction during indentation when the sample surface height variation is smaller than $15-\mu \mathrm{m}$ to help overcome this challenge. Measurements are made at room temperature, not $37^{\circ} \mathrm{C}$, so deviations in mechanical properties caused by this variation in temperature cannot be evaluated, though they would be expected to be minor. The influence of the underlying alveolar wall architecture on observed mechanical properties is difficult to determine with the current light microscopy setup. For instance, it would be desirable to determine if alveolar walls exhibit anisotropy and different mechanical properties when indented in directions aligned with or transverse to the plane of the wall. However, the current specimens are thick and the imaging system does not have 3D capabilities, hence it is not possible to determine the local alveolar wall orientation at each point of contact. Finally, the influence of cellular constituents on measured mechanical properties remains to be fully elucidated. In the methods detailed here, no efforts are made to specifically remove cellular constituents of the tissue. However, the cells present at the surface available for indentation are unlikely to be viable 
given the time elapsed since tissue harvest and the necessity of cutting the tissue to gain access to the alveoli. Specific experiments to remove cells, or repopulate matrices with viable cells, and evaluate the resulting changes in tissue stiffness would appear warranted.

Because fresh unfixed tissue is needed for these measurements, the time elapsed from tissue harvest to measurement should be minimized and samples should be stored at $4{ }^{\circ} \mathrm{C}$ to avoid changes in mechanical properties. Particular attention should be paid whenever tissue strips are transferred between containers during washing or staining so that minimum distortion or damage is generated. For AFM application in liquid, a crucial step is to cut the tissue as flat as possible and immobilize the sample on the supporting coverslip. If available, an automated sectioning machine such as a vibratome or tissue slicer can be used to cut slices of highly uniform thickness. It's important to attach tissue strips immediately before AFM measurements and minimize the time elapsed for AFM measurements as the sample will eventually detach from the coverslips. One useful observation is that larger strips appear to attach more stably to cover slips and remain in place for longer durations in PBS than smaller strips.

AFM microindentation can characterize samples spanning a broad range from $100 \mathrm{~Pa}$ to $50 \mathrm{kPa}$ (shear modulus) when using a standard 0.06 $\mathrm{N} / \mathrm{m}$ cantilever with a $5 \mu \mathrm{m}$ diameter spherical tip. This range can be expanded using probes with different spring constants; AFM probes with spherical glass tips ranging from 0.6 to $12 \mu \mathrm{m}$ in diameter and spring constants ranging from 0.01 to $0.58 \mathrm{~N} / \mathrm{m}$ are commercially available (e.g. Novascan) and commonly used ${ }^{3}$. With a $5 \mu \mathrm{m}$ spherical tip, the theoretical contact area between the tip and tissue is about $5-9 \mu \mathrm{m}^{2}$ for $400-700$ $\mathrm{nm}$ indentation (Fig. 1A). Smaller or larger tips can be used to provide smaller or larger scales of spatial resolution. Pyramidal tips have also been used in AFM microindentation ${ }^{13-16}$, providing smaller contact areas and thus increasing spatial resolution in mapping, though data fitting is more complex for this tip geometry.

Several limitations to this method should be noted. The lung has traditionally been mechanically characterized non-invasively, for instance using pressure-volume analysis ${ }^{17}$ or punch-indentation of whole lungs ${ }^{19,20}$. Invasive methods such as the one described here alter the lung architecture in important ways through the loss of the air-liquid interface that normally exists in the air-filled lung and the loss of pre-stress that maintains lung partial inflation upon relaxation of respiratory muscles. These limitations are common to all measurements made in lung tissue strips ${ }^{18}$. Notably, however, the median stiffness measured in the parenchyma of normal lung tissue (shear modulus $\sim 0.5 \mathrm{kPa}$ ) does not differ substantially from estimates based on punch-indentation of intact lungs at resting volumes ${ }^{19,20}$. While lung tissue is known to exhibit non-linear stiffening with increasing deformation, it is not possible to test in a rigorous fashion whether this property persists down to the micro-scale with the methods employed here. The Hertz model assumes homogeneity of the sample. However, most biological materials, including lung parenchyma, are increasingly heterogeneous at decreasing spatial scales. Heterogeneity of the sample can result in artifacts like variation of the Young's modulus depending on indentation depth, i.e. depending on the layer or component that is deforming. The heterogeneity in the xy-plane can be limited by carefully choosing the appropriate spherical tip size depending on the microstructure of the biomaterial as proposed by Dimitriadis EK et al. ${ }^{8}$ It is much more difficult to predict or correct the Hertz model error due to material heterogeneity in the z-direction. Azeloglu et al. recently proposed a hybrid computational model to characterize the elastic properties of heterogeneous substrate with discrete embedded inclusions ${ }^{21}$. Their new technique provides a potential means to calculate inclusion properties of heterogeneous materials overcoming the limitations of Hertzian analysis.

The Hertz model also assumes absolute elastic behavior, while biological materials typically display time-dependent viscoelastic behaviors. A full viscoelastic characterization of tissue can be obtained by varying the indentation velocities used. Importantly, previous macro-scale mechanical testing of normal and fibrotic lung tissue demonstrates weak frequency dependence of lung tissue mechanical properties, and preservation of the differences between normal and fibrotic tissue mechanical properties across all frequencies tested ${ }^{11}$. These findings strongly suggest that the measurement of mechanical properties using a single indentation velocity with AFM captures an essential aspect of the changes in tissue mechanical properties that accompany fibrosis.

The Poisson's ratio of 0.4 for lung tissue used in this work is from a macroscopic measurement ${ }^{9}$. Unfortunately, the Poisson's ratio at microscale and any changes under disease condition are not available in the literature. As alternatives to $E, E /\left(1-u^{2}\right)$ or $\left(1-u^{2}\right) / \pi E($ denoted the elastic constant $k)^{22}$ can be calculated from AFM microindentation and reported when the Poisson's ratio is unknown. For most biomaterials Poisson's ratio is in the range of 0.4 to 0.5 due to their high water content. Within the range $0.3-0.5$, the factor $1 /\left(1-u^{2}\right)$ varies only from $1.10-1.33$, such that reasonable variations in the Poisson's ratio exert only modest effects on the reported modulus. The increase in shear modulus that we report for fibrotic tissue relative to normal tissue is several fold in magnitude, implying that errors associated with variations in Poisson's ratio are minor relative to the changes in mechanical properties observed.

The actual algorithm and code that can be used for the analysis of force-displacement data is subject to the specific application condition and the subsequent characteristics of the diverse populations of force-displacement curves. If more sophisticated analysis is of interest, one may consult the work of Lin et al. ${ }^{23}$. The authors compiled a series of synergistic strategies into an algorithm that overcomes many of the complications that have previously impeded efforts to automate the fitting of Hertz models of indentation data.

Several other areas are available for further development and exploitation of this method. In cases where one is interested in visualizing the alveolar walls without antibody labeling, both elastin and collagen can be visualized from their autofluorescent signal in the green spectrum. On the other hand, better imaging, using either thinner tissue sections, 3D imaging techniques, or both, could enhance the ability to correlate tissue architecture with underlying mechanical properties. While the current methods allow staining and visualization of extracellular matrix components such as collagen and laminin, additional efforts could be aimed at staining cell surface markers to identify specific cell populations and to characterize the mechanical microenvironments in the vicinity of such populations. Alternatively, tissue could be harvested from mice expressing fluorescently-tagged lineage markers or cell specific proteins to pursue the same goal. Finally, the method detailed here appears well suited to characterizing other anatomical features in the lung, such as vessels which remodel in hypertension, and airways which remodel in asthma. Based on its current state of development and potential for further enhancement, AFM microindentation appears poised to yield valuable insights into the changes in tissue stiffness that accompany disease progression in the lung, and will no doubt be of value in characterizing spatial and temporal changes in the stiffness of a variety of other soft tissues. 


\section{Disclosures}

No conflicts of interest declared.

\section{Acknowledgements}

We thank A. Tager and B. Shea for their ongoing collaboration, and for providing the lung tissue used for demonstration purposes here. This work was supported by National Institutes of Health grant HL-092961. This work was performed in part at the Center for Nanoscale Systems (CNS), a member of the National Nanotechnology Infrastructure Network, which is supported by the National Science Foundation under NSF award ECS-0335765. CNS is part of the Faculty of Arts and Sciences at Harvard University.

\section{References}

1. Wang, H.B., Dembo, M., \& Wang, Y.L. Substrate flexibility regulates growth and apoptosis of normal but not transformed cells. Am J Physiol Cell Physiol. 279, C1345-1350 (2000).

2. Paszek, M.J., et al. Tensional homeostasis and the malignant phenotype. Cancer Cell. 8, 241-254 (2005).

3. Klein, E.A., et al. Cell-cycle control by physiological matrix elasticity and in vivo tissue stiffening. Curr. Biol. 19, 1511-1518 (2009).

4. Discher, D.E., Janmey, P., \& Wang, Y. L. Tissue cells feel and respond to the stiffness of their substrate. Science. 310, 1139-1143 (2005).

5. Bergner, A. \& Sanderson, M.J. Airway contractility and smooth muscle $\mathrm{Ca}(2+)$ signaling in lung slices from different mouse strains. $J$ Appl Physiol. 95, 1325-1332 (2003).

6. Thundat, T., Warmack, R.J., Chen, G.Y., \& Allison, D.P. Thermal and ambient-induced deflections of scanning force microscope cantilevers. Appl Phys Lett. 64, 2894-2896 (1994).

7. Mahaffy, R.E., Shih, C.K., MacKintosh, F.C., \& Kas, J. Scanning probe-based frequency-dependent microrheology of polymer gels and biological cells. Phys Rev Lett. 85, 880-883 (2000).

8. Dimitriadis, E.K., Horkay, F., Maresca, J., Kachar, B., \& Chadwick, R.S. Determination of elastic moduli of thin layers of soft material using the atomic force microscope. Biophys. J. 82, 2798-2810 (2002).

9. Butler, J.P., Nakamura, M., Sasaki, H., Sasaki, T., \& Takishima, T. Poissons' ratio of lung parenchyma and parenchymal interaction with bronchi. Jap. J. Physiol. 36, 91-106 (1986).

10. Liu, F., et al. Feedback amplification of fibrosis through matrix stiffening and COX-2 suppression. J Cell Biol. 190, 693-706 (2010).

11. Ebihara, T., Venkatesan, N., Tanaka, R., \& Ludwig, M.S. Changes in extracellular matrix and tissue viscoelasticity in bleomycin-induced lung fibrosis. Temporal aspects. Am J Respir Crit Care Med. 162, 1569-1576 (2000).

12. Dolhnikoff, M., Mauad, T., \& Ludwig, M.S. Extracellular matrix and oscillatory mechanics of rat lung parenchyma in bleomycin-induced fibrosis. Am J Respir Crit Care Med. 160, 1750-1757 (1999).

13. Rehfeldt, F., Engler, A.J., Eckhardt, A., Ahmed, F., \& Discher, D.E. Cell responses to the mechanochemical microenvironment--implications for regenerative medicine and drug delivery. Adv Drug Deliv Rev. 59, 1329-1339 (2007).

14. Berry, M.F., et al. Mesenchymal stem cell injection after myocardial infarction improves myocardial compliance. Am J Physiol Heart Circ Physiol. 290, H2196-2203 (2006).

15. Azeloglu, E.U., Bhattacharya, J., \& Costa, K.D. Atomic force microscope elastography reveals phenotypic differences in alveolar cell stiffness. J Appl Physiol. 105, 652-661 (2008).

16. Wagh, A.A., et al. Localized elasticity measured in epithelial cells migrating at a wound edge using atomic force microscopy. Am J Physiol Lung Cell Mol Physiol. 295, L54-60 (2008).

17. Martinez, F.J. \& Flaherty, K. Pulmonary function testing in idiopathic interstitial pneumonias. Proc Am Thorac Soc. 3, 315-321 (2006).

18. Cavalcante, F.S., et al. Mechanical interactions between collagen and proteoglycans: implications for the stability of lung tissue. $J$ Appl Physiol. 98, 672-679 (2005).

19. Hajji, M.A., Wilson, T.A., \& Lai-Fook, S.J. Improved measurements of shear modulus and pleural membrane tension of the lung. J Appl Physiol. 47, 175-181 (1979).

20. Lai-Fook, S.J., Wilson, T.A., Hyatt, R.E., \& Rodarte, J.R. Elastic constants of inflated lobes of dog lungs. J Appl Physiol. 40, 508-513 (1976).

21. Azeloglu, E.U., Kaushik, G., \& Costa, K.D. Developing a hybrid computational model of AFM indentation for analysis of mechanically heterogeneous samples. Conf Proc IEEE Eng Med Biol Soc., 4273-4276 (2009).

22. A-Hassan, E., et al. Relative microelastic mapping of living cells by atomic force microscopy. Biophys J. 74, 1564-1578 (1998).

23. Lin, D.C., Dimitriadis, E.K., \& Horkay, F. Robust strategies for automated AFM force curve analysis--I. Non-adhesive indentation of soft, inhomogeneous materials. J. Biomech. Eng. 129, 430-440 (2007). 